Hervé Breton
Université de Tours (France)

\section{Réciprocité des points de vue et réflexivité collective en VAE: quel paradigme pour la validation des savoirs expérientiels?}

Reciprocity of viewpoints and collective reflexivity when validating prior experience: What paradigm for the validation of experiential knowledge?

doi:10.18162/fp.2019.492
$\mathrm{R}$

ésumé

L'objet de la présente étude porte sur les processus réflexifs nécessaires à la formalisation des acquis de l'expérience et à leur validation dans le cadre de la quatrième voie d'accès à la certification en France. Nous cherchons ainsi à examiner, à partir d'une enquête qualitative conduite auprès de cinq candidats, la circulation des processus réflexifs et les dynamiques de réciprocité des points de vue dans le cadre du parcours VAE (validation des acquis de l'expérience) : narration de l'expérience, formalisation des savoirs, présentation du dossier aux membres du jury.

Certification, collectif, expérientiel, réflexivité, savoirs.

Abstract

The purpose of this study is to examine the reflexive processes required to formalize and validate prior experience in the context of the fourth path to certification in France. Thus, based on a qualitative research survey of five candidates, we look at the changes in reflexive processes and the reciprocity dynamics of viewpoints during the country's official process for validating prior experience (called Validation des Acquis de l'Expérience, or VAE): narration of experience, formalization of knowledge, and presentation of the file to jury members.

Keywords

Certification, collective, experiential, reflexivity, knowledge.
La validation des acquis de l'expérience (VAE) constitue depuis 2002 en France la quatrième voie d'accès aux certifications professionnelles. Elle permet de valider tout ou partie d'un diplôme, titre ou certificat, moyennant un travail de formalisation des savoirs expérientiels. Cela suppose pour les adultes qui s'y engagent d'accomplir un parcours narratif et réflexif : celui de l'examen du vécu, de sa mise en récit, puis de son analyse thématique afin de chercher des correspondances avec le référentiel de certification. L'objet de la présente étude porte sur les processus réflexifs nécessaires à la formalisation des acquis de l'expérience et à leur validation. Cela nous conduit à différencier les processus réflexifs qui accompagnent la mise en récit du vécu en vue d'un travail de thématisation, de ceux dont l'enjeu est de délibérer collectivement sur la validité des savoirs qui sont examinés dans les procédures VAE.

Les enjeux d'un examen des processus réflexifs à l'œuvre dans les parcours VAE sont concrètement posés dans la notice de Pineau (2009) intitulée "Savoirs et rapports aux savoirs » dans laquelle il propose d'inverser le sigle de la VAE en EAV (Expérience Acquis Validation). Cette inversion signale un renversement paradigmatique. Tandis que la formation initiale pense l'éducation à partir d'une somme de savoirs constitués, pour ce qui concerne la VAE, les savoirs acquis par la pratique (Denoyel, 2009) doivent être formalisés. Cela suppose ainsi pour les candidats de franchir des passages successifs : saisie réflexive de leur expérience, description de l'agir en situation, temporalisation du parcours de constitution des acquis, mise en récit dans un dossier, présentation de ce dernier auprès d'un jury. Le travail réflexif rendu nécessaire par la singularité de cette quatrième voie d'accès à la certification est donc très singulier. 


\section{Les savoirs expérientiels : acquisition tacite, intersubjectivité et réflexivité collective}

$\mathrm{Du}$ point de vue du candidat, les savoirs acquis lui sont quasiment transparents. Apprendre par l'expérience (Pineau, 1991), c'est en effet constituer des savoirs sans que les processus d'acquisition soient identifiés et notés par le sujet. En d'autres termes, le développement d'une capacité ne prend pas la forme d'une conscientisation des savoirs acquis, mais d'une confiance accrue dans l'agir, d'une extension des possibilités d'action en fonction de ce que nécessite les situations rencontrées (Billeter, 2002, 2012). L'appréhension de ces savoirs en tant qu'objets de pensée (Prior, 2002) suppose un travail réflexif par lequel le sujet se détourne des enjeux poursuivis au cours des actions qu'il engage (le quoi) pour devenir attentif aux procédés d'action, aux manières dont il s'y prend pour conduire l'action (le comment). Ce détournement du regard correspond précisément à un acte réflexif, une « conversion du regard » : «Ce déplacement du regard de la conscience attentionnelle de l'objet à l'acte de conscience qui m'y donne accès renvoie très précisément à une conversion de type réflexif, dans la mesure où je libère par là la structure d'acte du perçu, et non plus seulement le perçu comme objet : c'est là une version possible de l'activité de la réduction, méthode s'il en est de la philosophie phénoménologique » (Cefaï et Depraz, 2001). Autrement dit, les savoirs acquis par l'expérience doivent pour advenir thétiquement au sujet qui les détient, être saisis par des actes de type réflexif : ralentissement, détournement du regard, éveil de l'attention aux procédés d'action, examen de la pratique... (Breton, 2009, 2016).

Cette activité réflexive conduite par le sujet amorce un travail de formalisation des savoirs expérientiels pour le sujet lui-même. Elle relève d'un examen de soi (Foucault, 1980/2013) mobilisant un travail réflexif conduit à l'échelle individuelle. Les processus de validation mobilisent un travail réflexif collectif fondé sur les processus d'intersubjectivité et d'attention conjointe (Breton, 2015).

Les récits d'expérience et/ou récits des candidats explicitent et décrivent en effet les savoirs expérientiels à partir d'un point de vue individuel et singulier : lecture des situations rencontrées, description de pratiques situées, énonciation des principes d'actions, monstration des valeurs sur lesquelles se basent des manières de faire... La description située des pratiques objective le point de vue en première personne, et génère, lors de la présentation des récits aux membres du jury VAE, un travail réflexif interindividuel et collectif. C'est de cette activité réflexive «à l'échelle du collectif " que peuvent advenir les processus d'intercompréhension qui déterminent les manières d'appréhender et de juger collectivement les savoirs expérientiels mis au jour dans et par les récits. Nous procédons à leur étude en différenciant l'activité réflexive conduite par le candidat qui doit formaliser ses acquis via un travail narratif en première personne, de celui du jury dont les membres délibèrent en seconde et troisième personne, lors de la réception des récits de pratiques, au moment de la présentation par les candidats de leur dossier. Nous posons ici l'hypothèse que l'accomplissement du trajet VAE suppose de conjuguer différents points de vue : celui du candidat, celui des membres du jury, celui du certificateur (représenté par le référentiel). Nous étudions donc dans le présent article la dynamique de réciprocité de ces points de vue en cherchant à comprendre comment circulent les processus réflexifs individuels et collectifs.

Le problème de la réciprocité des points de vue a été posé par Thomas Nagel (1974) dans un article devenu célèbre, intitulé : "What is it like to be a bat? ». La référence peut apparaitre éloignée, voire saugrenue, face au propos qui nous occupe. Cependant, en interrogeant « l'effet que cela fait d'être une chauve-souris ", Nagel met au jour la difficulté de comprendre la manière dont se construit un mode d'existence et/ou une pratique, du point de vue d'autrui : 
Il est souvent possible d'envisager un autre point de vue que le sien propre, en sorte que la compréhension des faits de ce genre ne soit pas limitée à notre propre cas. En un certain sens, les faits phénoménologiques sont parfaitement objectifs : une personne peut savoir ou dire ce qu'est l'expérience de l'autre qualitativement. Ils sont subjectifs, cependant, au sens où même cette attribution objective d'expérience est possible seulement pour quelqu'un qui soit suffisamment semblable à l'objet de l'attribution pour être en mesure d'adopter son point de vue - pour comprendre l'attribution à la première personne aussi bien qu'à la troisième personne. (Nagel, 1974, p. 441-442)

La tension identifiée par Nagel entre la compréhension objective de l'expérience d'autrui et l'appréhension subjective de cette même expérience lorsqu'elle est éprouvée en première personne (Zahavi, 2008, 2015) traverse de part en part les dynamiques réflexives à l'œuvre dans les parcours de reconnaissance et de validation des acquis. L'étude de ces dynamiques peut s'intéresser aux processus réflexifs qui permettent le passage d'une appréhension des savoirs expérientiels en première personne à une compréhension distribuée, intersubjective et collective, des acquis constitués par le candidat VAE au cours de l'exercice de son métier.

\section{Problématique de l'étude et présentation de l'enquête}

Le travail réflexif qui s'amorce à partir de l'expérience du sujet peut être dite " en première personne ". Cela procède alors d'un mouvement de désincorporation du savoir durant lequel le vécu professionnel est pensé, réfléchi, thématisé, d'abord selon une manière signifiante pour soi, puis, selon une logique qui concorde avec les formes de réception prévues et attendues par l'institution certificatrice. À l'inverse, du point de vue du jury, instance consacrée par le certificateur, l'activité réflexive est censée être au service du processus de validation, et donc présupposée se déployer à partir de l'instrument fondateur de la certification : le référentiel. Cependant, et c'est l'une des raisons pour laquelle l'activité réflexive est si décisive dans les pratiques de jury, le référentiel ne peut se constituer en pièce unique de la procédure de validation. Les repères qu'il fournit doivent sans cesse être interprétés en fonction de la singularité des dossiers, de l'histoire des candidats, de l'expression de chacun d'entre eux. La validation en VAE ne se résume donc pas à une délibération à partir de critères formels. Les membres du jury qui relèvent du corps académique ou du « monde professionnel » forment un collectif de sujets vivants, disposant eux-mêmes d'une connaissance expérientielle des métiers désignés par le diplôme. Le jury est donc, de ce point de vue, un collectif impliqué dans une activité d'interprétation conjointe et partagée, qui peut évaluer selon des normes prescrites, mais qui doit surtout, spécialement dans le cadre de la VAE, « interpréter ensemble» la pratique narrée ${ }^{1}$.

La problématique de l'étude peut donc être résumée de la manière suivante : selon quels processus les acquis expérientiels d'un sujet deviennent-ils appréhendables pour un jury dans le cadre d'une procédure VAE? Afin d'examiner ces processus, nous avons procédé à des entretiens compréhensifs auprès de cinq adultes inscrits dans la VAE pour une certification de niveau 1 : le Certificat d'aptitude aux fonctions de directeur d'établissement ou de service d'intervention sociale (CAFDES) ${ }^{2}$. Nous avons ainsi cherché à examiner la circulation des processus réflexifs individuels et collectifs lors du parcours VAE, et notamment : ceux mobilisés par chacun des candidats lors de la phase d'écriture de leur dossier VAE; ceux mobilisés lors de la phase de présentation de ce dossier aux membres du jury. 


\section{Première phase de l'enquête : modélisation du parcours VAE}

Nous avons, pour la première phase de l'enquête (Figure 1), commencé par chercher à différencier les différentes phases du parcours VAE des candidats :

\begin{tabular}{cl} 
Phases & Séquence \\
\hline Phase 1 & Analyse du parcours, des emplois et recherche de correspondances avec les référentiels \\
\hline Phase 2 & Information sur la VAE et sélection de la certification \\
\hline Phase 3 & Inventaire de l'expérience et instruction du dossier de recevabilité \\
\hline Phase 4 & Formalisation de l'expérience par la rédaction du livret 2 \\
\hline Phase 5 & Présentation du dossier devant le jury VAE \\
\hline Phase 6 & Notification de la décision du jury et scénarios d'évolution \\
\hline
\end{tabular}

Figure 1

Le parcours VAE en 6 séquences.

Cette modélisation (Breton, 2016) permet de mettre au jour un paradoxe : le référentiel de certification pour la VAE est commun avec les trois autres voies d'accès (formation initiale, formation continue, apprentissage). La tension entre le caractère objectif des savoirs codifiés de la formation formelle et celui, subjectif, des savoirs acquis par l'expérience est donc présente dès la première étape du parcours. Cette configuration a pour effet de contraindre les candidats à définir une stratégie narrative (donc d'en forger la capacité) afin d'exprimer leur vécu professionnel et d'en analyser le contenu en cherchant à se rapprocher graduellement des attendus du référentiel (donc sans les perdre de vue). Le premier « réglage » à produire ne porte donc pas sur le contenu de l'expérience dont il faudrait rendre compte - par exemple, une situation professionnelle particulière permettant d'expliciter un pan de la pratique spécialement désigné par le référentiel de compétences ou de certification -, mais sur le choix des "procédés narratifs» les plus adéquats pour accompagner le passage d'une appréhension subjective des savoirs incorporés à une présentation formalisée de savoirs détenus et référençables. En clair, le candidat doit, avant de s'engager dans la narration d'un contenu expérientiel, décider du moyen narratif par lequel lesdits savoirs seront à terme le plus concrètement appréhendables par les membres de jury.

Un pan de l'étude a donc porté sur l'analyse des processus réflexifs et narratifs mis en œuvre par les candidats pour instruire leur dossier (figure 1 - phase 4 du parcours), l'objet de cette analyse étant de mettre au jour les formes de contraintes générées par la structure des dossiers sur les procédés narratifs des candidats engagés dans le travail de formalisation de leurs acquis. 


\section{Seconde phase de l'enquête : examen des contraintes narratives générées par la structure du « livret 2 »}

Le récit initie donc un travail réflexif durant lequel le candidat fait advenir son expérience au langage, saisit thétiquement les savoirs acquis au cours de l'exercice de son métier, narre les évolutions de ses manières de voir, d'agir et de s'impliquer dans l'exercice de son métier. La pratique narrative doit ainsi, dans les parcours VAE, rendre manifeste des savoirs acquis, au cours de l'expérience, dans le cours de la vie. En d'autres termes, c'est la mise en mots de l'expérience qui doit révéler les savoirs acquis tacitement, silencieusement, souvent à l'insu du sujet. Ce sont ces constats qui nous ont conduits à examiner et à chercher à comprendre les stratégies narratives mises en œuvre par les candidats pour instruire leur dossier.

Pour ce faire, nous avons étudié dans cinq dossiers de VAE CAFDES (le livret 2 qui est rédigé lors de la quatrième phase du parcours [figure 1]) les récits de pratiques dont la fonction est de manifester les savoirs et acquis expérientiels. Puis, nous avons collecté les écritures successives de situations rédigées pour chacun des cinq livrets 2 , afin de comprendre comment s'organisait, du point de vue des candidats, l'activité narrative et réflexive au cours de leur parcours VAE.

Un point mérite en effet d'être souligné d'entrée : les récits de pratiques - qui prennent la forme de récits d'expérience professionnelle - doivent respecter (dans les dossiers VAE attendus dans les procédures françaises) un format déterminé, un nombre limité de signes et de pages. Le candidat doit donc apprendre à exprimer et écrire ses savoirs et capacités dans un texte dont le format est contraint. Cette situation n'est pas fondamentalement exceptionnelle. Il existe en effet peu de contextes sociaux dans lesquels l'espace et le temps pour se dire ne sont pas comptés. Cependant, ce que nous souhaitons mettre en lumière ici, c'est que le format alloué pour les récits dans les dossiers VAE a un impact sur les modalités possibles de mise au jour des savoirs expérientiels. Pour ce qui est du diplôme CAFDES, terrain de la présente étude, le format prescrit pour la description de la pratique est de dix mille signes, espaces compris. Cette prescription contraint le candidat à régler ses procédés narratifs selon deux plans : étendre ou réduire la durée du vécu de référence à partir duquel va se déployer le récit; intensifier ou réduire le niveau de détail de la description de ce même vécu.

En d'autres termes, les contraintes de format du livret encadrent l'activité narrative des candidats et orientent vers un type de récit aux dépens d'autres. L'étude de la structure des livrets permet ainsi de mettre au jour la conception qu'ont les certificateurs de ce qu'est un savoir expérientiel, et de la manière jugée pertinente d'en rendre compte. Ce point peut être examiné à partir de la différenciation, parmi les pratiques narratives, entre récits de vie (Bertaux, 2005) et description des pratiques en situation. En ce qui concerne la perspective biographique, son apport pour la VAE peut se comprendre ainsi :la narration de la pratique au cours d'une période de vie est de nature à manifester des acquis expérientiels selon une perspective longitudinale. Cette pratique du récit présente différents avantages : elle permet de comprendre les modes de constitution, dans le temps, des manières de faire et d'exercer un métier; elle permet d'appréhender la façon dont un agir s'est transformé qualitativement dans la durée, en ancrant les processus d'apprentissage dans l'histoire du sujet. Le pendant de l'appréhension longitudinale des modes de constitution des acquis est le faible niveau de détail atteint dans la description des procédés. À l'inverse, le régime de la description permet, en réduisant drastiquement la durée du vécu de référence, 
de mettre en relief des gestes, de détailler des manières d'agir et de penser, de mettre en mots des inférences produites en contexte... En bref, la description permet «d'exemplifier » la pratique, en présentant dans les dossiers, des situations courtes, mais très détaillées.

L'étude de la structure des dossiers CAFDES nous amène à constater que le paradigme de l'analyse de l'activité (Barbier et Durand, 2017) est implicitement suggéré aux candidats pour rendre leurs savoirs manifestes. C'est d'ailleurs le paradigme dominant en France, pour ce qui concerne les procédures VAE. C'est ce qui explique le recours fréquent à l'entretien d'explicitation (Vermersch, 1994) pour accompagner le travail de mise au jour des acquis expérientiels. Il convient cependant de noter que l'importance donnée à ce registre descriptif dans les dossiers traduit (ou trahit) les théories des savoirs expérientiels sur lesquelles les certificateurs français se fondent pour construire leurs procédures. D'autres scénarios sont en effet possibles : solliciter des récits de type biographique, privilégier des approches longitudinales et historicisantes ${ }^{3}$, faire évoluer les référentiels de certification en intégrant de manière plus conséquente les logiques des compétences transverses ${ }^{4}$. L'expérience du Portugal ouvre, de ce point de vue, des pistes de travail intéressantes et prometteuses (Cavaco, 2013).

\section{Troisième phase : enquête sur les procédés de codification des savoirs professionnels}

Parmi les facteurs qui impactent l'activité réflexive des acteurs concernés et impliqués dans les parcours VAE, le référentiel occupe une place à part (Chauvigné, 2010). Il serait plus juste de parler " des référentiels " (de certification, de compétences, d'activités, d'emploi) et d'en analyser à la fois la spécificité et les liens qu'ils entretiennent avec l'expérience professionnelle et le réel du travail à partir duquel ils sont censés être élaborés. Pour des raisons pratiques, nous allons les penser ensemble, tel un édifice tenant différents aspects d'un même projet : le référencement des savoirs professionnels. Pour chacun des référentiels, les processus d'édification apparaissent en effet proches : la visée est d'aboutir à un document définissant des périmètres d'activités et de compétences ${ }^{5}$ par métier, selon une logique de typification des pratiques. De ce point de vue, les référentiels ont la curieuse particularité d'apparaître comme des instruments au service d'une lecture des situations de travail qui peut être nommée « radicalement en troisième personne ${ }^{6} »$. La moyennisation des pratiques à l'œuvre dans leur construction permet de désigner un très grand nombre de classes de situations. Elle fait émerger des situations typiques auxquelles, théoriquement, les vécus singuliers doivent pouvoir se référer. Cependant, à l'état pratique, les savoirs référencés adossés aux situations typiques construites pour et par les référentiels prennent l'aspect - du fait notamment du vocabulaire abstrait qui les nomme - de savoirs en troisième personne pour lesquels il n'y a justement plus personne, soit, pour lesquels plus aucune expérience singulière ne peut être concrètement appréhendée.

C'est pourtant en fonction du ou des référentiels que les acteurs impliqués dans les procédures et processus VAE sont censés s'orienter, réfléchir et délibérer. L'étude de la structure du référentiel du CAFDES (terrain de notre étude) permet d'identifier quatre activités types (AT) :

- Élaboration et conduite stratégique d'un projet d'établissement ou de service (AT1)

- Management et gestion des ressources humaines (AT2)

- Gestion économique, financière et logistique d'un établissement ou d'un service (AT3)

- Expertise de l'intervention sociale sur un territoire (AT4) 
Le candidat VAE doit donc, lors de la rédaction de son dossier, chercher à démontrer qu'il a acquis les savoirs nécessaires à la conduite de ces quatre activités. De ce point de vue, le référentiel définit le périmètre des activités du métier, énonce les compétences attendues pour l'exercer, voire spécifie le niveau de maîtrise attendu pour chacun des savoirs listés. Il constitue ainsi un document qui norme et prescrit. En ce qui concerne la France, le système national des certifications date de $1969^{7}$. Ce système hiérarchise en cinq niveaux l'ensemble des certifications, en les étalonnant du niveau $V$ au niveau I. Un décret paru en 1972 a ensuite institué les Commissions professionnelles consultatives (CPC) dont l'une des missions est d'élaborer les référentiels. Puis, la loi de 2002 - fondatrice pour la VAE - créera le Répertoire national des certifications professionnelles (RNCP) qui rend nécessaire, pour qu'une certification puisse y être référencée, d'avoir formalisé des référentiels et d'avoir structuré une procédure VAE.

L'édification des systèmes de certification est donc codépendante de la production de référentiels dont la fonction est de formaliser des normes. Le rôle des CPC est précisément d'examiner collectivement le travail réel des praticiens, afin d'en rendre compte dans un premier référentiel (le référentiel d'activités). L'élaboration de celui-ci (il peut parfois être précédé d'un référentiel large : le référentiel d'emploi) permet de poursuivre la démarche en interrogeant les ressources nécessaires à la conduite des activités précédemment identifiées. Cette seconde étape vise l'élaboration du référentiel de compétences (Tableau 1 ci-dessous). Cette étape franchie, un troisième référentiel peut alors être élaboré : le référentiel de certification qui, lui, prescrit les modes d'évaluation et de validation (donc les critères) sur lesquels doivent se fonder les actes d'attribution de la certification.

\section{Tableau 1}

CNCP - Distinction présentée entre référentiel d'activités et référentiel de certification ${ }^{8}$

\begin{tabular}{|c|c|c|c|c|}
\hline \multicolumn{2}{|c|}{ Référentiel d'activités } & \multicolumn{2}{c|}{ Référentiel de certification } \\
\hline Activités et tâches & $\begin{array}{c}\text { Compétences associées aux } \\
\text { activités et aux tâches }\end{array}$ & $\begin{array}{c}\text { Compétences ou capacités qui } \\
\text { seront évaluées }\end{array}$ & $\begin{array}{c}\text { Modalités } \\
\text { d'évaluation }\end{array}$ & $\begin{array}{c}\text { Critères } \\
\text { d'évaluation }\end{array}$ \\
\hline
\end{tabular}

La logique d'édification des référentiels, généralement pilotée par le certificateur, et conduite avec les CPC, mobilise un travail collectif et réflexif dont l'enjeu est d'édifier un document commun. Par l'analyse du travail avec les professionnels, le réel du travail examiné est traduit en activités types et sous-activités, auxquelles s'accrochent des savoirs et des compétences (Figure 2). 


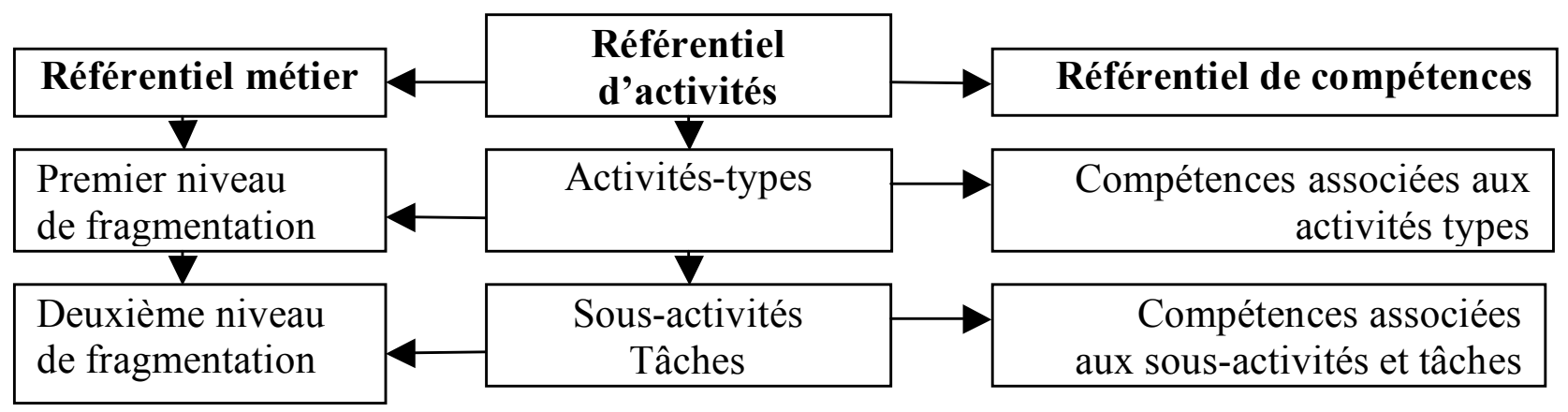

Figure 2

Mise en référentiel et niveau de fragmentation des activités types (AT) des métiers.

Le résultat est cependant, par certains aspects, discutable. En effet, outre les biais de construction résultant des transactions entre les groupes de professionnels, représentants des centres de formation et du certificateur au sein de la $\mathrm{CPC}^{9}$, la mise en référentiel du métier mobilise des logiques et des ingénieries qui fragmentent et dévitalisent les pratiques. Stéphane Balas (2016, p. 2) identifie dans le processus de conception des référentiels une triple euphémisation : « euphémisation liée à une analyse au mieux partielle de ce travail, euphémisation en rapport au dialogue social qui se noue autour de la production de ce référentiel et brouille les frontières entre travail et emploi (Jobert, 2013 ${ }^{10}$ ) ou encore euphémisation due à l'intégration du diplôme produit dans un système éducatif préexistant, impliquant des contraintes formelles fortes. Dès lors, le produit final est souvent très éloigné du travail réel. » C'est pourtant à partir des référentiels que les membres du jury sont tenus de délibérer collectivement. Dans les faits, les référentiels, s'ils définissent un périmètre à la délibération, en identifiant notamment les activités types et les compétences, semblent peu propices au travail collectif d'interprétation. En effet, ils cantonnent les pratiques de délibération à des critères formels qui dessinent une représentation typifiée des situations de travail. En d'autres termes, la recherche d'une correspondance exacte entre les savoirs expérientiels mis en récit dans les livrets du candidat et les savoirs codifiés des référentiels génère des modes scolaires d'évaluation et des processus de délibération fondés sur le contrôle des connaissances. C'est par l'activité réflexive collective que cette impasse est en partie évitée lors des sessions de jury VAE.

\section{Saisie réflexive du vécu et formalisation des acquis : analyse des procédés}

Cette étude a été conduite, nous l'avons dit, auprès de cinq candidats VAE. Nous choisissons cependant de nous centrer, pour rendre compte de manière concrète des procédés à l'œuvre, de présenter les données recueillies auprès d'un seul candidat (un homme de 50 ans, directeur d'une association d'économie sociale et solidaire depuis une quinzaine d'années, titulaire lors de l'entrée dans le parcours VAE d'un diplôme de niveau IV) engagé dans un parcours VAE CAFDES. Nous avons cherché à comprendre les processus réflexifs à l'œuvre au cours de l'activité narrative dont la visée est de formaliser ses acquis dans le cadre de l'écriture de son dossier (le livret 2). Pour cela, nous avons analysé les transcriptions résultant d'entretiens compréhensifs (Kaufmann, 1996) et d'explicitation (Vermersch, 1994), puis étudié les écritures successives qui ont été nécessaires pour la rédaction de son dossier. 
Nous présentons dans cette section deux passages extraits de son Livret de présentation des acquis de l'expérience (Livret 2) :

Première écriture (juin 2017) de la situation de travail : Définition et conduite d'un projet d'établissement ou de service à visée stratégique ou opératoire [CAFDES - Livret de présentation des acquis de l'expérience - Section 4.2.1]

«Le projet d'établissement étant un processus déjà en œuvre qui évolue,je pratique l'évaluation interne à l'aide de tableaux de bord de suivi des activités. Je transmets les résultats trimestriels au personnel en réunions de services et sollicite sa participation aux réglages à apporter pour améliorer le service. Je pratique le même mode participatif en conseil d'administration. L'évaluation qualitative et quantitative des résultats sert de base de travail au CA pour proposer des orientations en phase avec le projet d'établissement, l'évolution des besoins des bénéficiaires et les attentes des financeurs...»

$--$

Troisième écriture ${ }^{11}$ (septembre 2017) de la situation de travail: Définition et conduite d'un projet d'établissement ou de service à visée stratégique ou opératoire [CAFDES - Livret de présentation des acquis de l'expérience - Section 4.2.1]

\section{PROJET D'ÉTABLISSEMENT}

a. Constitution de groupes de travail et validation par étapes

Pour définir collégialement le projet d'établissement, je propose une méthode basée sur l'implication volontaire des usagers, des salariés et des administrateurs (suite...)

b. Mise en cuvre et évaluation

L'actualisation et l'animation du projet d'établissement génèrent la refonte d'une délégation de pouvoir (cf. annexe 3 p. 52) qui permet de nommer et de dissocier les rôles, les missions, les pouvoirs et responsabilités respectives du directeur (ma fonction) et du président (personne morale) (suite...)

c. Méthodologie et évaluation interne

Depuis 1999, je pratique une méthodologie de projet (suite...)

d. Évaluation externe

N'ayant pas de demande d'agrément à effectuer, je n'ai pas recours à une évaluation... (suite...)

e. Démarche qualité en cours

Avec le support de fiches de procédures (suite...)

L'analyse de ces deux passages permet d'appréhender la transformation des registres narratifs qui s'opèrent, au cours de l'écriture, et qui peuvent être décrits de la manière suivante : l'analyse réflexive de l'expérience s'amorce au cours de la première écriture à partir d'un récit, en première personne, impliqué et situé. L'action est décrite de manière chronologique, et le vocabulaire mobilisé est personnel 
et singulier. En d'autres termes, l'activité narrative s'organise en mobilisant des cadres temporels et un registre de vocabulaire d'abord signifiant du point de vue du sujet qui a vécu l'expérience décrite. Puis, graduellement, et c'est ce qui peut être observé dans le second passage (septembre 2017), le candidat reconstruit son récit en prenant en compte, le point de vue du lecteur, des membres du jury, et, à travers eux, celui du certificateur. Ce travail de reconstruction mobilise des actes réflexifs qui procèdent d'un changement de regard, ce qui rend possible de s'apercevoir que les savoirs peuvent être considérés et nommés selon différents points de vue. C'est ce travail réflexif qui permet le remaniement du texte : la forme narrative impliquée (en première personne) s'estompe au profit d'une présentation thématique; le vocabulaire mobilisé devient compatible avec celui utilisé dans les référentiels. La stratégie narrative du candidat évolue donc au gré des écritures successives, et passe ainsi d'une écriture impliquée à celle d'une écriture thématique, qui cherche à s'accorder avec les logiques et le vocabulaire prescrits du référentiel CAFDES.

L'analyse de l'activité narrative conduite par le candidat montre qu'il réalise un travail de décentrement de type réflexif: son attention, d'abord portée sur la description en première personne de sa situation, intègre graduellement au cours de l'activité narrative, la variété des manières d'appréhender les savoirs mis en forme dans le premier récit. L'acte réflexif procède ici d'une « conversion du regard " qui se traduit par la prise en compte des différentes manières de dire, de nommer, de décrire et de narrer les savoirs expérientiels. Cette conversion n'est cependant ni statique ni irréversible. Elle est dynamique, réversible et réciproque : elle peut adopter différents points de vue pour appréhender et objectiver les savoirs expérientiels : celui du sujet impliqué qu'il est; celui des membres du jury qui sont pour partie des professionnels et professionnelles du métier; celui du certificateur, qui impose ses vues au travers du référentiel. La saisie réflexive des «écarts de point de vue " peut ici être considérée comme déterminante pour la définition des stratégies narratives à privilégier.

\section{Procédés mobilisés lors de l'audition : réflexivité collective et collectif d'interprétants}

Nous voyons ici toute la complexité de la situation de validation, dont la responsabilité revient aux membres de jurys $\operatorname{VAE}^{12}$. Sans que cela soit toujours remarqué (Cortessis, 2014), la discussion (phase 5 de la figure 2) s'entame en effet sur un malentendu relatif : du point de vue du candidat, les savoirs expérientiels sont des savoirs vivants, incarnés, évolutifs, circonstanciels; du point de vue des membres du jury, les mêmes savoirs peuvent apparaître, s'ils ont les yeux rivés sur le référentiel, génériques (point de vue académique) et/ou typiques (point de vue des référentiels professionnels). La distribution des points de vue varie donc potentiellement entre le singulier, le typique et le général (Quéré, 2000), les modes d'expression de ces trois registres correspondant aux trois formes d'expression : en première, seconde et troisième personne. Le travail réflexif à l'œuvre entre les acteurs du parcours VAE peut donc être analysé, selon une dynamique collective, à partir de la circulation des modes d'appréhension des savoirs. La complexité du processus provient ici du fait que ces variations génèrent des transformations dans les manières de voir, de définir et de dire les savoirs. Le registre du générique mobilise en effet un vocabulaire définissant des classes d'actions très éloignées des manières de dire observables dans les situations réelles de travail (Jobert, 2015). À l'inverse, le registre du singulier mobilisé par le candidat sollicite des formes d'expression sous la forme d'un langage dont la signification est d'abord individuelle et locale. De plus, et cela ajoute à la complexité du processus, les manières de dire vont varier selon 
les échelles de temps à partir desquelles s'édifie la narration du vécu. Face au vocabulaire intemporel mobilisé dans les référentiels, les modes de narration permettant la mise au jour des savoirs mobilisés dans la pratique sont éminemment temporalisés, inscrits dans des situations éprouvées, et dans un devenir qui n'est autre que l'histoire du sujet.

L'étude du vécu de l'audition du candidat VAE CAFDES dont nous avons présenté deux passages extraits de son livret 2 montre que le travail d'écriture et de remaniement successif des différentes versions en vue de permettre la circulation des manières d'appréhender ses savoirs expérientiels a permis de préparer et d'accompagner la délibération collective. La stratégie narrative retenue pour l'écriture, qui faisait place au registre impliqué de la première personne, en intégrant des dimensions biographiques dans d'autres sections du dossier (notamment la Section 2.1 - Votre parcours professionnel et la Section 2.2 - Vos activités bénévoles) et en variant dans les registres d'expression entre le descriptif (registre de la première personne) et le thématique (registre de la troisième personne), a été de nature à ouvrir un espace de dialogue sur ses acquis, sans restreindre la discussion aux seuls critères formels qui sont prescrits par le référentiel. Concrètement, les actes réflexifs permettant la circulation des manières d'appréhender et de narrer les savoirs expérientiels mobilisés lors de la rédaction du livret 2 semblent avoir été réitérés lors de la phase d'audition. En d'autres termes, cette capacité acquise par l'écriture du dossier semble avoir été une ressource pour l'accompagnement de l'activité réflexive des membres du jury, afin qu'ils envisagent les savoirs selon différents types de repères, de critères et de normes. L'activité réflexive collective en situation de validation des savoirs expérientiels peut ainsi se définir, à partir de l'exemple présenté, comme un travail par lequel le collectif mobilise des actes qui lui permettent d'adopter le point de vue singulier du candidat (première personne), les points de vue qui circulent au cours des échanges (deuxième personne), le point de vue formel des savoirs codifiés du référentiel (troisième personne).

\section{Synthèse et perspectives}

L'étude des stratégies mises en œuvre par les cinq candidats VAE CAFDES de la présente étude a cherché à comprendre la manière dont ils avaient défini une ligne de conduite pour écrire leur dossier. Le second plan de l'enquête a porté sur les stratégies de présentation de ce dossier lors de l'audition. L'échantillon étant restreint, les résultats avancés ici ne peuvent être généralisés. Cependant, nous pouvons remarquer que parmi les cinq candidats impliqués dans cette étude, ceux qui ont obtenu le meilleur taux de validation ont trouvé les moyens, au cours de la description écrite et orale de leur parcours et de leur pratique, d'accompagner les processus réflexifs du collectif «jury » afin qu'une circulation s'opère dans les manières d'appréhender, de penser et de juger de la validité des savoirs expérientiels présentés lors de l'audition. La stratégie retenue a été de faire émerger des processus d'intercompréhension en variant à la fois les formes de narration (descriptive, narrative, biographique) permettant l'expression des savoirs constitués par l'expérience, et les registres de vocabulaire (première, deuxième, troisième personne) permettant de les nommer et de les désigner.

Ces éléments soulignent l'importance du maintien d'une éthique de la discussion (Habermas, 2013) lors de la phase d'échange avec le jury. Plus qu'une opération arithmétique s'organisant par addition des points de vue de chacun de ces membres, l'activité délibérative apparaît dépendante de la capacité réflexive du collectif qui permet de penser et d'interpréter ensemble les récits présents dans le dossier et le discours de chacun des candidats. 


\section{Notes}

1 L'étude présentée n'interroge pas les procédures qui organisent des sessions de validation des acquis à partir de la mise en situation.

2 Il s'agit du Certificat d'aptitude aux fonctions de directeur d'établissement ou de service d'intervention sociale (CAFDES), diplôme de niveau 1 (Système national de certification français [nomenclature de 69]), proposé par la Direction générale de la cohésion sociale. http://www.rncp.cncp.gouv.fr/grand-public/visualisationFiche?format=fr\&fiche=367

3 L'analyse de la structure des dossiers proposés par les certificateurs montre que c'est le paradigme de l'analyse de l'activité qui s'est imposé comme méthode aux candidats pour la formalisation de leurs acquis. Cela peut sembler cohérent d'un certain point de vue (familier de la troisième personne) puisque le même paradigme est à l'œuvre dans les travaux conduits par les CPC.

4 Nous soulignons ici les recherches très contemporaines sur le paradigme des compétences transverses, et les résultats produits par la recherche Eure.k (www.eure-k.eu) intitulée : Valider et certifier les compétences clés européennes.

5 Nous utiliserons pour cette étude les termes de «savoirs expérientiels », en les pensant comme ontologiquement distincts des savoirs référencés. Suivant cette voie, la compétence peut être comprise comme la donnée nécessaire à la construction des certifications. Pour une appréhension de la notion de compétence et de ses limites, le lecteur pourra consulter l'article de Batal et Fernagu-Oudet (2013).

6 Nous proposons de comprendre cette expression de la manière suivante : la posture « radicalement en troisième personne » est celle où l'expérience ne se réfère à personne de particulier ni à aucune expérience singulière. Il s'agit donc du régime spéculatif de la pensée.

7 La note de 2007 du Centre d'études et de recherches sur les qualifications (CEREQ) intitulée « La construction de l'Europe de la compétence. Réflexions à partir de l'expérience française » (Collection BREF, n 244) pourra être ici consultée : http://www.cereq.fr/publications/Cereq-Bref/La-construction-de-1-Europe-de-la-competence.-Reflexions-apartir-de-1-experience-francaise.

8 Ce tableau est extrait du dossier formalisant la procédure d'enregistrement d'une certification telle que préconisée par la Commission nationale de la certification professionnelle (CNCP). Le dossier est téléchargeable sur le site de la CNCP : http://www.cncp.gouv.fr/enregistrement-de-droit.

9 Sur ce sujet, le lecteur pourra consulter l'étude présentée par le CEREQ (2012, Net.Doc nº 89), intitulée « Place et rôle des professionnels dans la conception des diplômes professionnels » : http://www.cereq.fr/content/download/4009/38843/file/Net-doc-89.pdf.

10 Jobert, G. (2013). Le formateur d'adultes : un agent de développement. Nouvelle revue de psychosociologie, (15), 31-44. http://dx.doi.org/10.3917/nrp.015.0031

11 Nous ne présentons pas ici de passages relatifs à la seconde écriture qui a été produite au cours du mois de juillet 2017. Son examen permet cependant de constater les transformations graduelles de l'écrit du fait du travail de thématisation en cours.

12 Les règles de composition d'un jury VAE en France sont les suivantes : respect d'une stricte proportion parmi les membres entre représentants du monde professionnel et du monde académique. En ce qui concerne l'effectif, il faut noter des variations importantes, selon les certificateurs (ministères et branches professionnelles).

\section{Références}

Balas, S. (2016). Comment concevoir des référentiels de diplômes professionnels sans renoncer au travail réel? Activités, 13(2). http://dx.doi.org/10.4000/activites.2889

Barbier, J.-M. et Durant, M. (dir.). (2017). Encyclopédie d'analyse des activités. Paris : Presses universitaires de France.

Batal, C. et Fernagu-Oudet, S. (2013). Compétences : un folk concept en difficulté? Savoirs, (33), 39-60. http://dx.doi.org/10.3917/savo.033.0039 
Bertaux, D. (2005). Le récit de vie ( $2^{\mathrm{e}}$ éd.). Paris : Armand Colin.

Billeter, J. F. (2002). Leçons sur Tchouang-Tseu. Paris : Allia.

Billeter, J. F. (2012). Un paradigme (2 éd.). Paris : Allia.

Breton, H. (2009). De l'échange intentionnel à l'analyse attentionnelle des pratiques. Se professionnaliser par le développement de la capacité réflexive. Dans C. Guillaumin, S. Pesce et N. Denoyel (dir.), Pratiques réflexives en formation : ingéniosité et ingénieries émergentes (p. 57-65). Paris : L'Harmattan.

Breton, H. (2015). Co-explicitation, attention conjointe et fonctions d'accompagnement en formation. Éducation permanente, (205), 87-98.

Breton, H. (2016). Attentionnalité émancipatoire et pratiques d'accompagnement en VAE. Recherches et éducations, (16), 51-63.

Cavaco, C. (2013). Reconnaissance et validation des acquis de l'expérience. Les résultats (in)attendus. Questions vives, 10(20), 79-93. http://dx.doi.org/10.4000/questionsvives. 1383

Cefaï, D. et Depraz, N. (2001). De la méthode phénoménologique dans la démarche ethnométhodologique. Dans M. de Fornel, R. Ogien et L. Quéré (dir.), L’ethnométhodologie (2éd., p. 99-119). Paris : La Découverte.

Chauvigné, C. (2010). Les référentiels en formation. Des normes en confrontation. Recherche et formation, (64), 77-90. http://dx.doi.org/10.4000/rechercheformation.210

Cortessis, S. (2014). La VAE, comme épreuve d'argumentation continue. Recherches É éducations, (10), 95-116. Repéré à https://journals.openedition.org/rechercheseducations/1897

Denoyel, N. (2009). Savoirs pratiques et VAE. Dans J.-P. Boutinet (dir.). L'ABC de la VAE (p. 212-214). Paris : Érès.

Foucault, M. (1980/2013). L'origine de l'herméneutique de soi. Paris : Vrin.

Habermas, J. (2013). De l'éthique de la discussion. Paris : Flammarion.

Jobert, G. (2015). Travail et créativité : catachrèses et vicariances. Éducation permanente, (202), 9-15.

Kaufmann, J.-C. (1996). L'entretien compréhensif. Paris : Armand Colin.

Nagel, T. (1974). What is it like to be a bat? The Philosophical Review, 83(4), 435-450. Repéré à https://warwick.ac.uk/fac/cross_fac/iatl/study/ugmodules/humananimalstudies/lectures/32/nagel_bat.pdf

Pineau, G. (1991). Formation expérientielle et théorie tripolaire de la formation. Dans G. Pineau et B. Courtois (dir.). La formation expérientielle des adultes (p. 29-40). Paris : La documentation française.

Pineau, G. (2009). Savoirs et rapports aux savoirs. Dans J.-P. Boutinet (dir.), L'ABC de la VAE (p. 210-211). Paris : Érès.

Prior, A. (2002). Objets de pensée. Paris : Vrin.

Quéré, L. (2000). Singularité et intelligibilité de l'action. Dans Centre de recherche sur la formation du Conservatoire national des arts et métiers (dir.), L'analyse de la singularité de l'action (p.147-170). Paris : Presses universitaires de France. http://dx.doi.org/10.3917/puf.derec.2000.01.0147

Vermersch, P. (1994). L'entretien d'explicitation. Paris : ESF.

Zahavi, D. (2008). Subjectivity and selfhood. Investigating the first-person perspective. Cambridge, MA : MIT Press.

Zahavi, D. (2015). Intentionnalité et phénoménalité. Un regard phénoménologique sur le "problème difficile ». Philosophie, (124), 80-104. http://dx.doi.org/10.3917/philo.124.0080

\section{Pour citer cet article}

Breton, H. (2019). Réciprocité des points de vue et réflexivité collective en VAE : quel paradigme pour la validation des savoirs expérientiels? Formation et profession, 27(2), 19-31. http://dx.doi.org/10.18162/fp.2019.492 